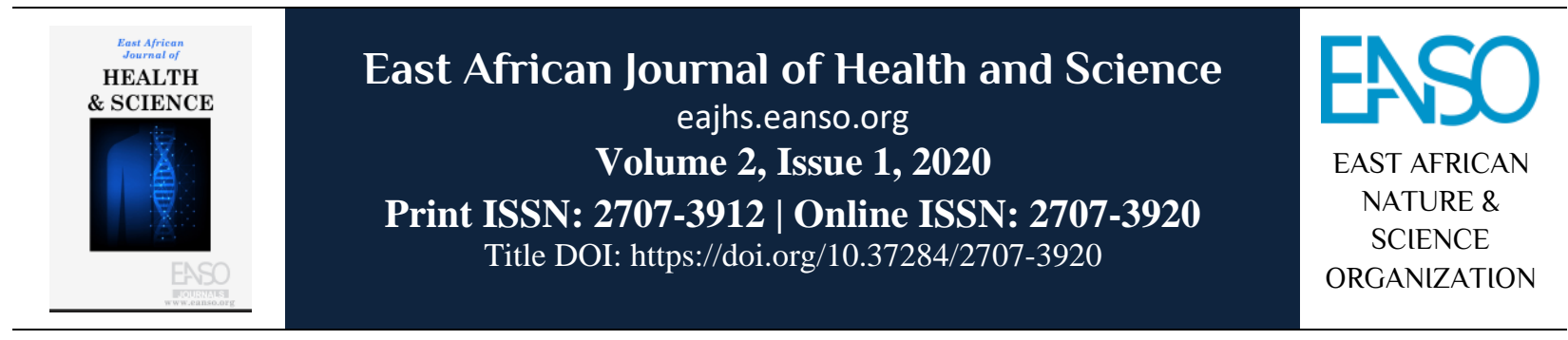

Original Article

\title{
The Effect of eHealth on Information Awareness on Non-Communicable Diseases Among Youths Between 18-25 Years in Nairobi County, Kenya, 2019
}

\author{
Stephen Ogweno $^{1,2 *} \&$ Dr. Eliphas Gitonga ${ }^{1}$ \\ ${ }^{1}$ Department of Population Health, School of Public Health, Kenyatta University, Nairobi, Kenya. \\ ${ }^{2}$ Stowelink Inc., Nairobi, Kenya. \\ *Author for correspondence email: stowelink@gmail.com. \\ ORCID: https://orcid.org/0000-0001-6727-192X
}

Article DOI: https://doi.org/10.37284/eajhs.2.1.136

\section{Date Published: ABSTRACT}

13 Apr 2020 Non-Communicable Diseases (NCDs) kill 41 million people each year, equivalent to $71 \%$ of all deaths globally while in Kenya $31 \%$ of deaths in the country in 2015 ,

Keywords: were due to NCDs. Majority of the populations dying from non-communicable

eHealth, diseases with over $85 \%$ are from developing countries. NCDs are also the leading

mHealth,

MyHeart-Ke App,

Non-Communicable

Diseases, cause of all hospital admission death-causing $51 \%$ of all hospital deaths in Kenya in 2015. The study sought to determine the impact of eHealth (healthcare practice supported by electronic processes) on non-communicable diseases information awareness levels in Nairobi County with the study population being the youths of Cardiovascular

Diseases, Kenyatta University. The research used a cohort study design where youths were STEPS survey, exposed to MyHeart-Ke App (a mobile app developed for this study giving information on NCDS and cardio-vascular health). They were then followed over six months to assess their knowledge on non-communicable diseases and healthy
lifestyle habits. A total of 402 respondents participated in the study. The study used posttest non-experimental cohort study design as there was only one study group. Data was collected using questionnaires. The majority of the study participants were males at $56 \%$ while the majority age was 22 years at $17 \%$. The study found out that awareness on NCDs among the respondents was high at $96 \%$ with cardiovascular diseases being the most widely known at $85.8 \%$. Although some of the participants knew m-Health at $68.7 \%$, it was not widespread among the respondents before the study. However, after the study, MyHeart-Ke was reported to offer a significant and relevant amount of information on NCDs with $51.2 \%$ preferring it over the mainstream internet. A majority also had a perception 
that NCDs are preventable through the reduction of alcohol use, screening, and healthy diets. The findings are expected to help the relevant authorities in designing appropriate strategies for addressing NCDs in the country especially in the digital space.

\section{APA CITATION}

Ogweno, S., \& Gitonga, E. (2020). The Effect of eHealth on Information Awareness on Non-Communicable Diseases Among Youths Between 18-25 Years in Nairobi County, Kenya, 2019. East African Journal of Health and Science, 2(1), 15-29. https://doi.org/10.37284/eajhs.2.1.136.

\section{CHICAGO CITATION}

Ogweno, Stephen, and Eliphas Gitonga. 2020. "The Effect of EHealth on Information Awareness on Non-Communicable Diseases Among Youths Between 18-25 Years in Nairobi County, Kenya, 2019”. East African Journal of Health and Science 2 (1), 15-29. https://doi.org/10.37284/eajhs.2.1.136.

\section{HARVARD CITATION}

Ogweno, S. and Gitonga, E. (2020) "The Effect of eHealth on Information Awareness on Non-Communicable Diseases Among Youths Between 18-25 Years in Nairobi County, Kenya, 2019”, East African Journal of Health and Science, 2(1), pp. 15-29. doi: 10.37284/eajhs.2.1.136.

\section{IEEE CITATION}

S. Ogweno and E. Gitonga, "The Effect of eHealth on Information Awareness on Non-Communicable Diseases Among Youths Between 18-25 Years in Nairobi County, Kenya, 2019”, EAJHS, vol. 2, no. 1, pp. 15-29, Apr. 2020.

\section{MLA CITATION}

Ogweno, Stephen, and Eliphas Gitonga. "The Effect of EHealth on Information Awareness on Non-Communicable Diseases Among Youths Between 18-25 Years in Nairobi County, Kenya, 2019”. East African Journal of Health and Science, Vol. 2, no. 1, Apr. 2020, pp. 15-29, doi:10.37284/eajhs.2.1.136.

\section{INTRODUCTION}

Non-communicable diseases (NCDs) are the leading killer diseases globally (Ahern et al., 2006). Majority of the populations dying from noncommunicable diseases with over $85 \%$ of the deaths globally are in developing countries (Boulos et al., 2014). There are various measures by states to reduce the prevalence of NCDs (Silva et al., 2015). Some of the measures apply modern technology to create awareness among the population. With the advancement in technology globally a lot of opportunities and amazing progress has happened in the medical sector and with the use of eHealth countries like Japan and United States of America have had amazing growth in improving the health care of the population (Lewis \& Wyatt, 2014; Reynolds, 2017).

In Africa, according to the World Health Organization (WHO), non-communicable diseases form up to $50 \%$ of adult deaths with more cases of deaths being on the younger populations under the age of 45 years (Neumann, 2014). Africa is projected to have a $27 \%$ increase in noncommunicable diseases cases over the next ten years (Njoroge et al., 2017). Most of these cases are in sub-Saharan Africa, which is projected to have the highest incidence (Njoroge et al., 2017). In Kenya according to the Kenya Demographic and Health Survey (KDHS) 2014 and the STEPS Survey (This is the tool used to collect data and measure non communicable disease (NCD) risk factors within the World Health Organization STEPwise approach to surveillance), over $55 \%$ of the deaths in the country now are caused by NCDs, yet little effort is put in place to address the problem (Silva et al., 2015). Kenya's population has 83\% smartphones coverage making it the most connected country in Africa. The coverage presents an excellent opportunity for eHealth to solve the problem of NCDs (Lee et al., 2017).

The youth in Kenya has been neglected in as far as health education, especially surrounding the noncommunicable diseases (Gorski et al., 2016). The statement can be supported by the fact that $80 \%$ of deaths from non-communicable diseases occur prematurely in youths between ages 30- 70 years 
(Lewis \& Wyatt, 2014). The problem has led to increased cases of non-communicable diseases in Kenya, which majorly start in early life. As a result, addressing non-infectious diseases is very crucial in this day and time. According to Consumer Insight research in Kenya in 2013, 2 out of 3 Youth in Kenya now either own a mobile device or have access to one. With the use of eHealth, we can be able to get to the youth and even more get to the world at large with relevant information on NCDs.

With the increase in technology, mHealth (practice of medicine and public health supported by mobile devices.) has become a key player in securing the future of health care, and that is why Stowelink decided to develop MyHeart-Ke mobile application. The application contains all the relevant information on cardiovascular health and healthy living habits, which aim at reducing the incidence rates of non-communicable diseases. The application advocates for robust lifestyle adaptation to increase awareness of non-communicable diseases. The application is easy to understand and has precise information. The research project, therefore, aimed at establishing and identifying the effect of e-Health on the level of awareness on noncommunicable diseases among the youths between 18-25 years in Nairobi County, Kenya.

\section{Problem Statement}

Two-thirds of premature deaths in adults are related to childhood conditions and behaviour related to NCDs risk factors is common in teenagers. Over a hundred and fifty million young people smoke. Further, $80 \%$ of the adolescents do not get enough physical activity, $7 \%$ of adolescents partake in serious episodic drinking, and 41 million youth are overweight (WHO "NCD and Youth", 2016). The probability of dying at a young age from an NCDs in Kenya is currently at $18 \%$ and these numbers continue to rise (WHO, 2015). Despite the fact that there is evidence to prove that a lot of deaths in Kenya have occurred due to NCDs, little effort has been put in place to curb the situation (Ahern et al., 2006). There is also little innovation around the NCDs and as a result, more people continue to live with little knowledge about the diseases (Neumann, 2014). Youth in Nairobi County are exposed to all the 5 major risk factors to non-communicable diseases including, tobacco and alcohol abuse, lack of physical activity, and poor diets and environmental risk factors (Njoroge et al., 2017). The study, therefore, sought to measure the effect of eHealth on awareness levels of noncommunicable diseases among the youth in Nairobi County with our study population being the youth of Kenyatta University.

\section{Justification of the Study}

The study is very important especially at this period where the country is experiencing rapid advancements in technology and the rapid increase in NCDs among youths and younger generations in Kenya. The study is vital because the results obtained will be able to influence the innovation space with evidence-based results to advance on the use of eHealth in addressing NCDs. Though the results obtained in the study we will be able to address the problem of NCDs and prevent it before it becomes a national problem. Also, through the study, we can be able to collaborate with other likeminded individuals and organizations both in the public and the private sector and be able to influence policies and laws.

\section{Research Questions}

1.Can e-Health have an effect on information awareness on non-communicable diseases among youths between 18-25 years in Nairobi County, Kenya?

2. What is the perception of youth on noncommunicable diseases among youths between 18-25 years in Nairobi County, Kenya?

3. What is the influence of MyHeart-Ke mobile app on awareness levels on NCDs among youths between 18-25 years in Nairobi County, Kenya?

4. What is the uptake of MyHeart-Ke mobile application use among youths between 18-25 years in Nairobi County, Kenya?

\section{Null Hypothesis}

$\mathrm{H}_{0} 1$ : There is no relation between mHealth use and non-communicable diseases awareness 


\section{Objectives of the Study}

\section{Broad Objective}

To determine whether the use of mHealth will be able to influence lifestyle habits in order to prevent NCDs among youths between 18-25 years in Nairobi County, Kenya.

\section{Specific Objectives}

1.To determine the extent of non-communicable diseases awareness among youths between 18-25 years in Nairobi County, Kenya

2.To determine the perception of noncommunicable diseases among youths between 18-25 years in Nairobi County, Kenya

3. To determine the influence MyHeart-Ke mobile application usage on the awareness levels among youths between 18-25 years in Nairobi County, Kenya

4. To determine the uptake of MyHeart-Ke mobile application on the youth as a means of learning about health care among youths between 18-25 years in Nairobi County, Kenya

\section{Significance and Anticipated Output}

The research will be of great significance in the innovation in the health care space and also in the global health space. The research findings will be used to influence and reinforce innovation and creativity in the health space. The findings will also encourage the development of eHealth technologies and creation of more simplistic and well-framed apps which will be used to communicate messages on NCDs. Through this project and the innovations that arise from it, we will be able to achieve sustainable development goal 3 i.e. Good Health and Wellbeing and more importantly help spearhead the achievement of the Big 4 Agenda.

\section{Limitations of the Study}

The study faced limitations such as ignorance by the respondents. Ignorance led to the lack of the needed information among the respondents on the mobile application. To address the challenge, the researcher did a user education at the beginning of the study to ensure all the study population starts from an even platform equipped with the right information on how to use and interact with MyHeart-Ke mobile app.

\section{LITERATURE REVIEW}

\section{Beginning of eHealth 1900s}

The use of technology for health began way early before the boom came about in the 2000s (Buijink et al., (2013). With the development of the radios in the 1900s, a revolution was felt (Silva et al., 2015). Doctors wondered whether they could use this to expand their health reach to patients further away. In 1940 the very first radiology images were sent 38 kilometres away using radio frequencies becoming the first use of eHealth (Breen \& Matusitz, 2010). During this period eHealth was used for mental health patients. It is also evident that back then eHealth began with focusing on mental health which would later be classified under non-communicable diseases (Njoroge et al., 2017).

\section{eHealth in the 2000s Era (2000-2005)}

In the United States of America, because of the rise of NCDs and chronic conditions, it was becoming increasingly difficult to keep up with patient management and upkeep in the hospitals (Ahern et al., 2006). Having identified this problem, The Robert Wood Johnson Foundation in 2002 set up a nationwide programme named the 'Health etechnologies Initiative' in order to find ways in which they could leverage on the existing technologies (Boulos et al., 2014). Later, they wanted to ensure that there was efficiency and improved health quality at the time. The move created one of the first applications of modern Health in the world (Ahern, 2006). With the rapid advancement in technology and the introduction of broadband internet, more rapid approaches were used by doctors and clinicians to try and improve quality of health care (Njoroge et al., 2017). In 2005, the World Health Organization commissioned key priority areas for advancements of eHealth which saw the upscaling of eHealth practices globally (Buijink et al., 2013). 


\section{Mobile Health (e-Health) Leading to m-Health}

Mobile health (mHealth) rose from eHealth due to the rapid advancements in technologies and the development of short message service platforms, android phone operating systems, and iPhone operating systems (Silva et al., 2015). These new advancements led to more opportunities for the interaction of health and technology. With more complex phones, mobile applications were developed where people could learn and interact with their physicians being miles away thus revolutionizing forever the field of mHealth (Boulos \& Brewer, 2014; Mburu \& Oboko, 2018). By 2010, it was estimated that there had been already 200 million downloads of health-related mobile apps and it was estimated that by 2017 there were about 1.7 billion (Silva \& Rodrigues, 2015).

\section{Mobile Health in Kenya}

According to Oxford Business Group, in their recent survey, mHealth had penetrated the country reaching across to both the rich and the poor (Maina, 2019). As a result, improving access to health care in Kenya (Lewis \& Wyatt, 2014). Kenya is noted as a front runner in Eastern Africa in terms of health care access and provision due to improved access and coordination of mHealth and traditional medicine (Kenya Healthcare Federation, 2006). The Ministry of Health in a bid to support eHealth in Kenya launched the first eHealth policy guidelines in 2011. Since then, the information and communications technology departments in collaboration with the Ministry of Health have harnessed this opportunity (Boulos et al., 2014).

The mHealth projects in the country have been implemented with a focus on HIV/AIDS and NCDs as the strategic focus areas (Njoroge et al., 2017). Kenya has done exceptionally well in these areas. However, little evaluation has been done on these initiatives. There has been very little input in Kenya as afar as mHealth for NCDs prevention is involved. Furthermore, there has been even lesser contribution of mHealth with a target on the youth (Ahern et al., 2006). Fafanuka platform is the most recent development in Kenya by Safaricom which uses short message service (SMS) platform to communicate information on non-communicable diseases prevention launched in September 2017 (Eunice, 2017, p. Daily Nation).

\section{Summary of the Literature and Gaps}

It is evident that eHealth has come a long way and has been used as a global alternative to enhancing the quality of health care and the effectiveness of health care. The rise of mHealth has even made it better to address health issues with proven global impact. In Kenya, it's evident that the healthcare sector has adapted the technology in health with much focus on NCDs and epidemics like HIV/AIDS. The field of non-communicable diseases has been neglected for a long time in Kenya in as far as eHealth and mHealth technologies are concerned despite the fact that it is the leading killer disease group globally and in the country. However, recent advancements to focus on non-communicable diseases have started to grow with mHealth technology of Fafanuka from Safaricom being the latest nationwide innovation in as far as mHealth and non-communicable diseases are concerned. Despite the rise of Fafanuka platform, it is still very blurry on efforts that have been put across to focus on preventive aspects of non-communicable diseases and the youth. Little effort has been placed on the youth presenting a gap of opportunity for research and innovation to solve this problem and address this big knowledge deficit.).

\section{MATERIALS AND METHODS}

\section{Research Design}

The study used a cohort study design. The design allowed the researcher to understand the effect of the intervention, in this case, MyHeart-Ke mobile application. The study design also allowed for a post-test on the study subjects, as a result, the impact of the intervention can be measured. The study used posttest non-experimental design meaning there was only be one study group which went through the posttest studies. The study maintained to be a cohort study since with the assumption that before the release of MyHeart-Ke app, nobody in the study population had access to the app. The research did not test the awareness levels on non-communicable diseases and cardiovascular health among the students before the 
intervention of the e-Health because according to a very recent study done in Kenya, (Shiroya, Neuhann, Müller, \& Deckert, 2019) they found out that Kenyans have very low awareness levels on what NCDs are and as a result conducting that primary data collection would have not been an effective use of the limited resources that were available for this study. The participants of the study used the app for 6 months after which a posttest was carried out to determine the influence of the app on the users' habits.

\section{Variables of the Study}

The dependent variables in this study were the awareness levels of non-communicable diseases and cardiovascular health as the study was focusing on this issue. The use of MyHeart-Ke mobile application was the independent variable as its usage among the study population which influence the awareness levels on NCDs.

\section{Location of the Study}

The study was carried out in Nairobi County, Kenya. This being the capital of the country represents the national outlook of the population. Also, this population is ideal for the study as they have the most access to mobile phones, internet and are actively engaged in interactions with their phones. Also, the assumption was that this population is predisposed to all the major risk factors of the NCDs and the awareness was low.

\section{Study Population}

The study populations were youths aged 18- 25 years old at Kenyatta University. The study population was ideal for the research as they have access to internet, courtesy of free Wi-Fi connection. Also, the study population is situated in Nairobi County which has the most risk to the 5 major leading risk factors to NCDs including tobacco use, alcohol use, physical inactivity and poor diets and environmental factors. These five factors also strongly reflected in the youth at Kenyatta University making the study population ideal for the study.

\section{Inclusion and Exclusion Criteria}

\section{Inclusion Criteria}

The inclusion criteria were anyone aged between ages 18-26 years old. The respondent was also required to own a mobile or a smartphone, agree to be part of the study, and be able to read, write and understand.

\section{Exclusion Criteria}

The following are the characteristics in participants which will bar them from participating in the study. Those who were critically ill and mentally challenged individuals.

\section{Sampling Method}

The study used a multi-stage sampling method. Purposive sampling was used to select Kenyatta University since it has a significant number of youths. Purposive sampling was also used to select youths who had mobile phones. Simple random sampling was then used to select the individuals who participated in the study.

\section{Sample Size Determination}

At any given point in Kenyatta University's the biggest schools i.e. School of Education and School of Economics have an estimate of 1350 students at the main campus respectively. According to the Yamane formula, the study selected a sample size of $30 \%$ of the total population. Therefore,

$30 \%$ of $1350=450$ youths.

Out of these 1350 youth per school, the research intended to have a confidence level of $95 \%$ and a margin of error of $5 \%$. The sample size was thus, calculated using the

Yamane method. According to the formula;

$\mathrm{N}=$ sample size

$\mathrm{E}=$ error levels

$\mathrm{N} /\left(1+\mathrm{Ne}^{2}\right)=1350$

$(1+1350(0.052)=1350$

$(1+1350(0.0025)=201.2422$

201 people per school. 
Since the study used two schools, School of Education and School of Business the sample size therefore was

$201 \times 2=402$ youths.

The participants were selected by the nonprobability sampling technique using convenience sampling as the key method for obtaining individuals from these 2 major schools. The procedure was used because it was the most efficient for pilot testing of the MyHeart-Ke app.

\section{Research Instruments}

The study involved the use of an online questionnaire developed by the researcher in close collaboration with the Kenyatta University School of Public Health and Applied Human Sciences. The online questionnaire was constructed with Google Forms making it readily available to all participants in the study. The questionnaire had four parts including the informed concept form and the bio data segments, information on non-communicable diseases, participant's views on mHealth and finally the participants' views on MyHeart Ke app. MyHeart Ke app that was used during the study was also designed by the research team led by Ogweno Stephen and was available on Playstore for easy access by the study participants. All these members were trained on cardiovascular health at the beginning of the study and they all used MyHeart$\mathrm{Ke}$ App for 6 months. The questionnaires were administered once at the end of the project.

\section{Validity and Reliability}

\section{Validity}

The validity was achieved through pretests. The pretests were done in Kenyatta university Ruiru campus under the assumption that it has the same characteristics as the study population of the study which is Kenyatta university. The pretests enabled the researcher to assess the tools and ensure that they were suited to collect the data. Validity was also attained through a random selection of study participants.

\section{Reliability}

Reliability of the data collected for the study was achieved through the supervision of data collection exercise. The keen supervision ensured that the collected information was authentic.

\section{Data Collection Techniques}

Data was collected via administration of questionnaires on Google Forms.

\section{Administering Questionnaires}

Questionnaires were administered through an online platform to all the subjects. The method was ideal for the study since the participants are active users of their mobile phones. They also have access to a mobile phone and/or a laptop as well as access to free internet. The procedure, therefore, made data collection from online forms easy, convenient, time-saving, and affordable.

\section{Data Analysis}

Online collected data from the surveys was analyzed using SPSS and Microsoft Excel software. The data was analyzed using percentage differences and correlation. The analysis was done offline with key insights on whether the research achieved its objectives being derived from the analysis.

\section{Logistical and Ethical Considerations}

This research followed the highest ethical standards and respect for human rights and freedom. The participation was voluntary and informed consent was obtained. The participants were also informed regarding the procedures of the research project and as a result, they signed a written consent form. Confidentiality and anonymity of the subjects were protected and maintained during the study. The respondents were also informed of the purpose of research beforehand and that they are well informed before they begin participating in the research. Permission was sought from the relevant authorities prior to the study. 


\section{RESULTS}

\section{Socio-demographics of the Respondents}

A total of 402 participants participated in the study which took 9 months. A majority of the respondents at $72(18 \%)$ were aged 22 years while the least at $32(8 \%)$ were aged 25 years. The data is as shown in Figure 1 below.

Figure 1: Age of the Respondents

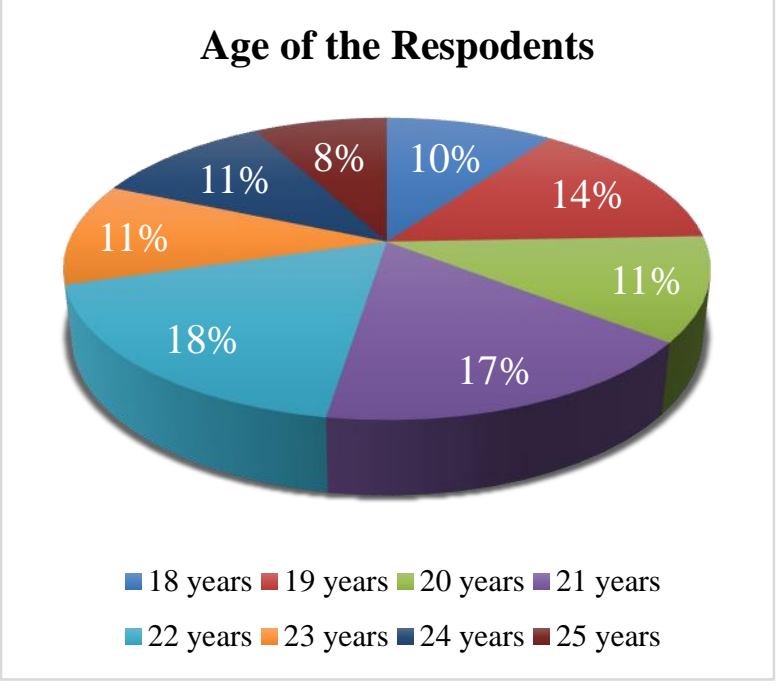

The majority of the study participants at $225(56 \%)$ were males while the least at $177(44 \%)$ were females. The data is as shown in Figure 2 below.

Figure 2: Gender of the Respondents

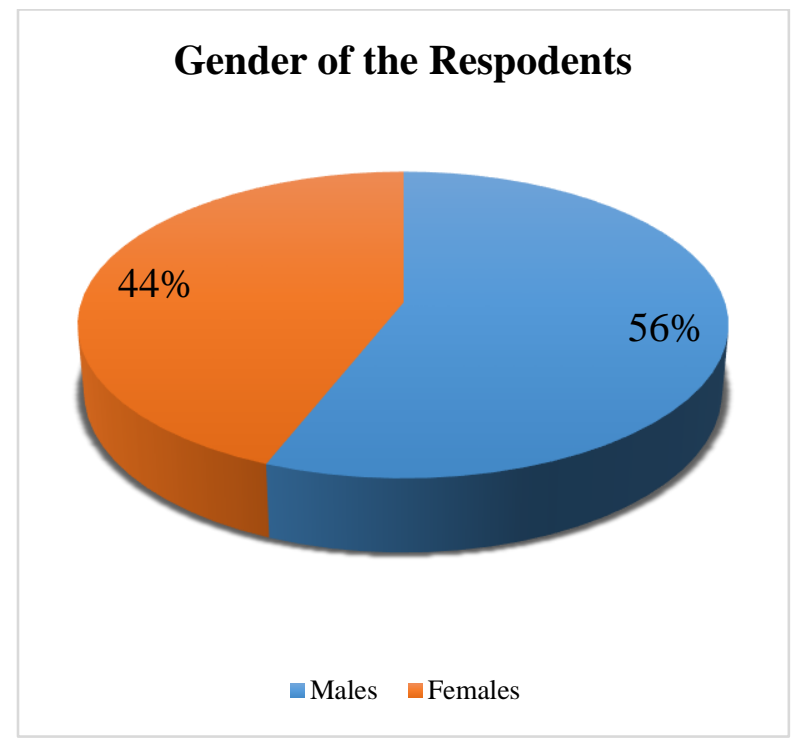

\section{Awareness Level among the Youths on Non- Communicable Diseases}

On the awareness of NCDs, a majority at $386(96 \%)$ were knowledgeable while a minority at $6(4 \%)$ did not know what the diseases are. The data is as shown in Figure 3 on the next page.

Figure 3: Respondents Knew Non-Communicable Diseases

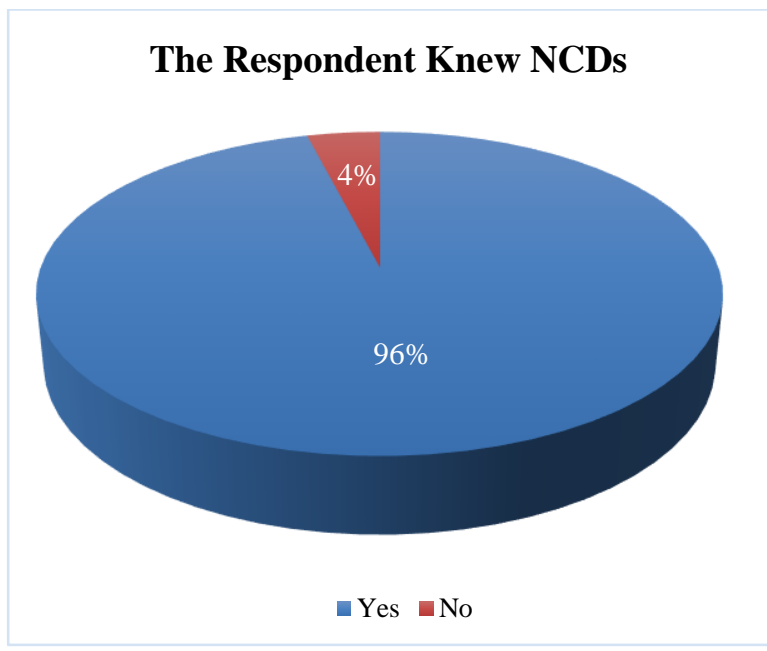

On the type of NCDs that the respondent knew, the study showed that cardiovascular diseases are the most known at $85.8 \%$ while other conditions like obesity, mental illness kidney failures, asthma, high blood pressure, atherosclerosis, arthritis, osteoporosis, Alzheimer's diseases, typhoid, and cholera had only $0.2 \%$ awareness levels.

The inferential statistics indicated that there was a significant association between the age of the respondent and information awareness on noncommunicable diseases

$(\chi 2=34.187, d f=1, p=0.001)$.

The results are as summarized in Table 1 below

Table 1: Socio-demographics of the respondents in relation to the Information awareness on non-communicable diseases

\begin{tabular}{llcl}
\hline $\begin{array}{l}\text { Socio- } \\
\text { demographics }\end{array}$ & $\chi^{2}$ & df & p-value \\
\hline Age & 34.187 & 1 & $0.001^{*}$ \\
Gender & 17.339 & 3 & 0.354 \\
\hline$(* \mathrm{p} \leq 0.05$ is significant at $95 \% \mathrm{Cl})$ &
\end{tabular}


East African Journal of Health and Science, Volume 2, Issue 1, 2020

Article DOI: https://doi.org/10.37284/eajhs.2.1.136

\section{Perception of the Youth towards Non- Communicable Diseases}

The study deemed to highlight what are the different views and understandings that the participants had especially around noncommunicable diseases. A majority at $89.8 \%$ of the youth knew what risk factors caused non- communicable diseases with only $5 \%$ being unaware of what the risk factors were.

Three hundred and twenty [320 (79.6\%)] of the participants recognized tobacco smoking as the major risk factors to developing non-communicable diseases while genetic factors, stress and salt intake were only reported by $1 \%$ of the respondents. The data is as demonstrated in Figure 4 below.

Figure 4: Perception on the Risk Factors

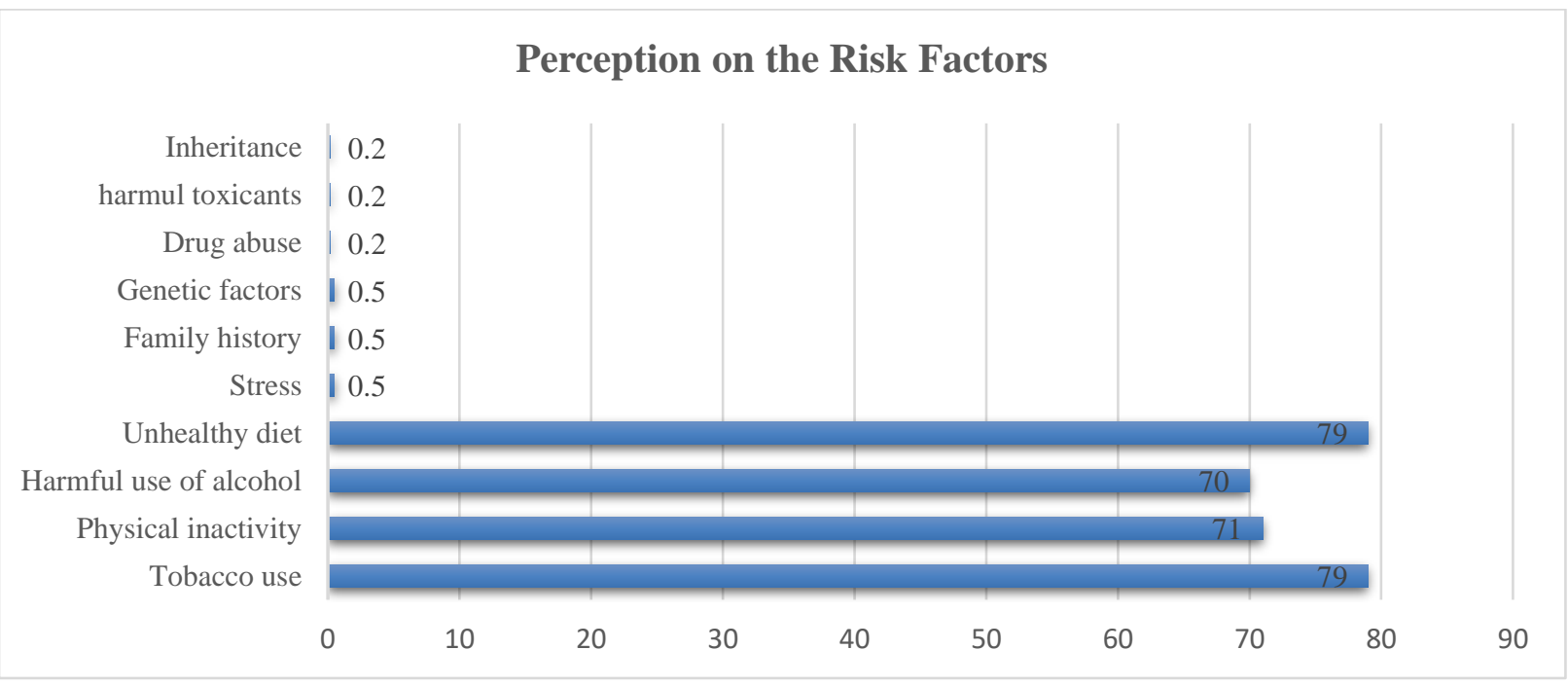

A majority at $94.5 \%$ agreed that non-communicable diseases could be prevented and over $70 \%$ of the participants thought that: early screening, focusing on healthy diets, reducing tobacco use and alcohol intake and reducing on salt intake could in fact help reduce non-communicable diseases. Sixteen [16 $(4 \%)$ ] of the respondents also believed that reducing stress was one way in which we could prevent non-communicable diseases. An equal of the respondents at $318(79 \%)$ indicated that screening and healthy diet was a major way of reducing NCDs. The data is as summarized in Figure 5 below.

Figure 5: Perception on How Non-Communicable Diseases can be prevented

\section{Perception on How NCDs can be Prevented}

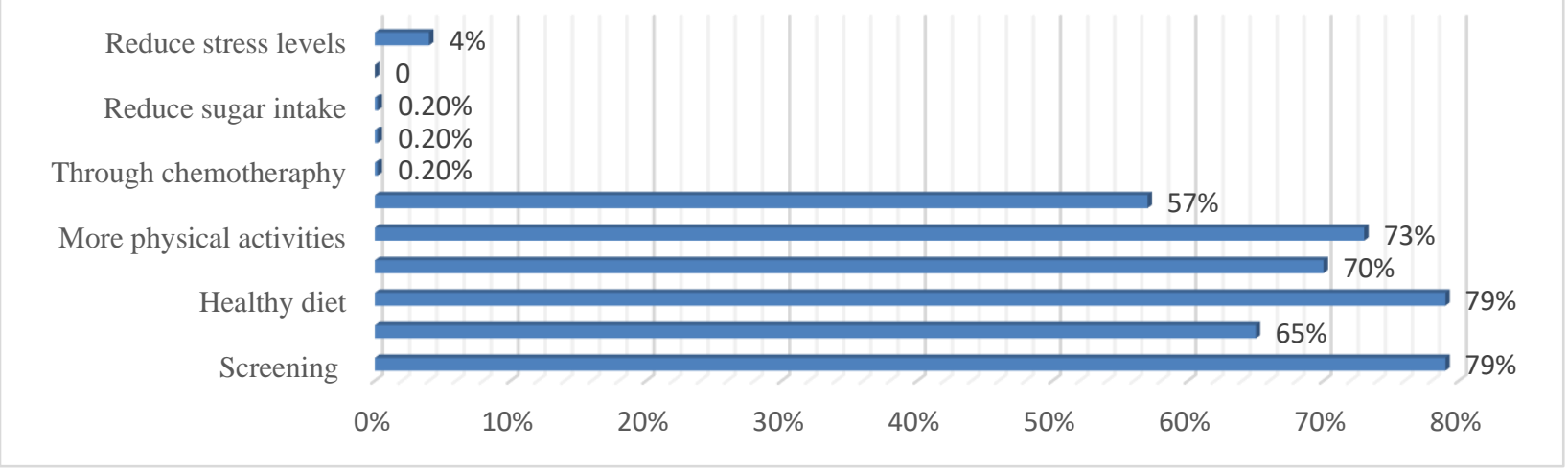


It was noted that $75.4 \%$ of the participants learned and heard about NCDs first while they were in school while $2 \%$ had the information first from personal research, from Stowelink projects and through individual interest. Also, a significant 10\% attributed had their first knowledge on NCDs from TV. This is represented in Figure 6 below.

Figure 6: Source of Information on NonCommunicable Diseases

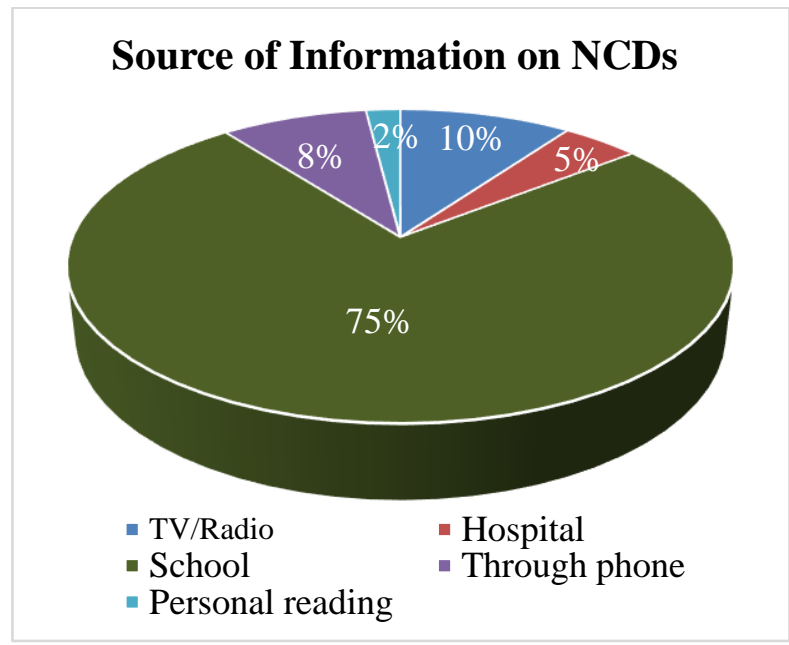

The perception on NCDs was measured using a 5point Likert scale. A majority of the participants at $196(48.8 \%)$ thought non-communicable diseases is a problem now as shown in Figure 7 and 169 $(42.2 \%)$ thought that this would be a problem in the future as shown in Figure 8.

Figure 7: Non-Communicable Diseases are a Problem in the Country Right Now

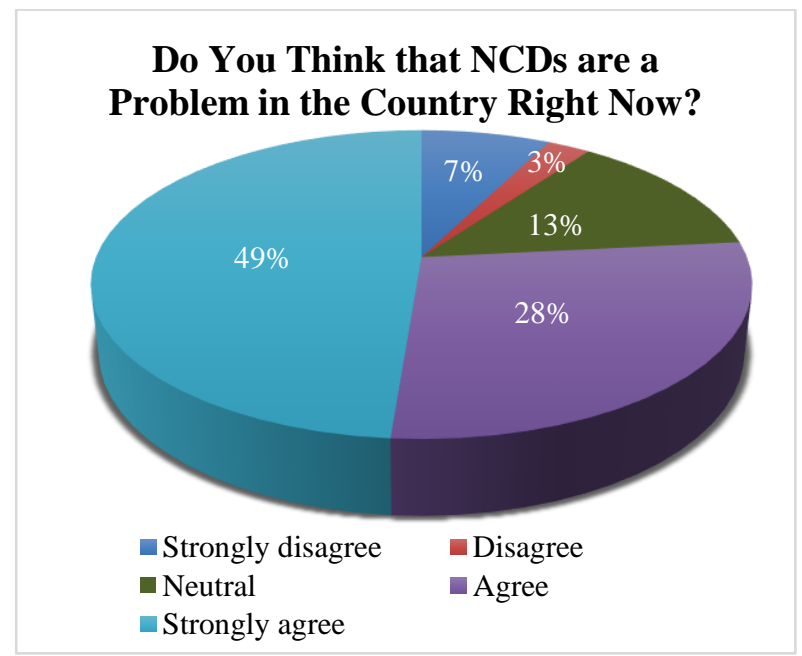

Figure 8: Non-Communicable Diseases Will be a Problem in Five Years

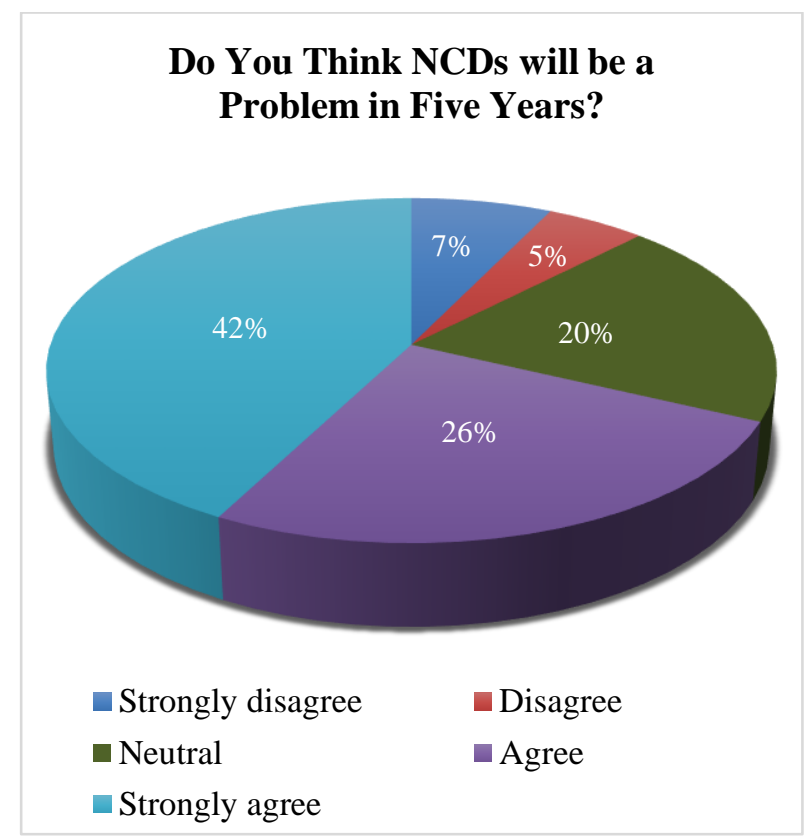

There was a general agreement on noncommunicable diseases being a problem among the youth with only $64(16 \%)$ of the total participants thinking it is not a problem as shown in Figure 9. In the old aged above 60, it was generally agreed that non-communicable diseases are a problem among them as represented in Figure 10.

Figure 9: Non-Communicable Diseases are a Problem in the Youth (14-26 years)

\section{NCDs are a Problem in the Youth (14-26 Years)}

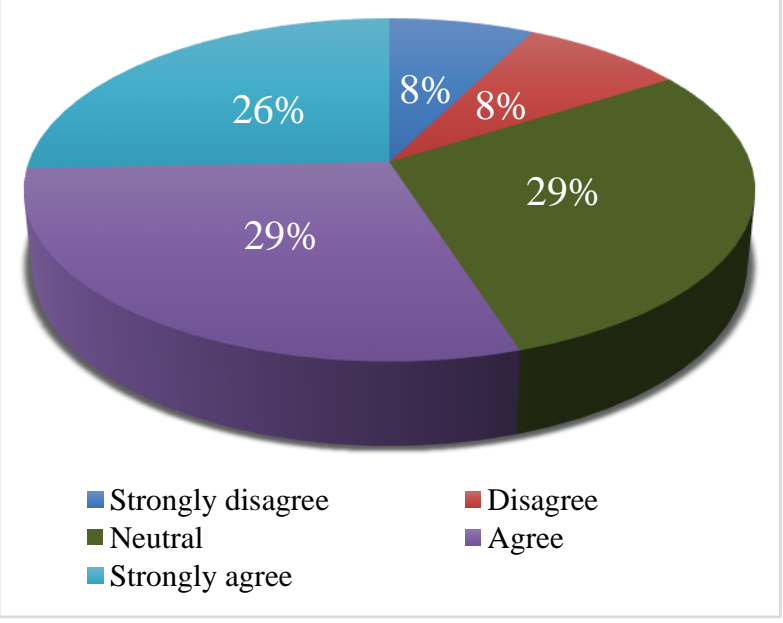


Figure 10: Non-Communicable Diseases are a Problem in Older People (Above 60 Years)

\section{NCDs are a Problem in Older People} (Above 60 Years)

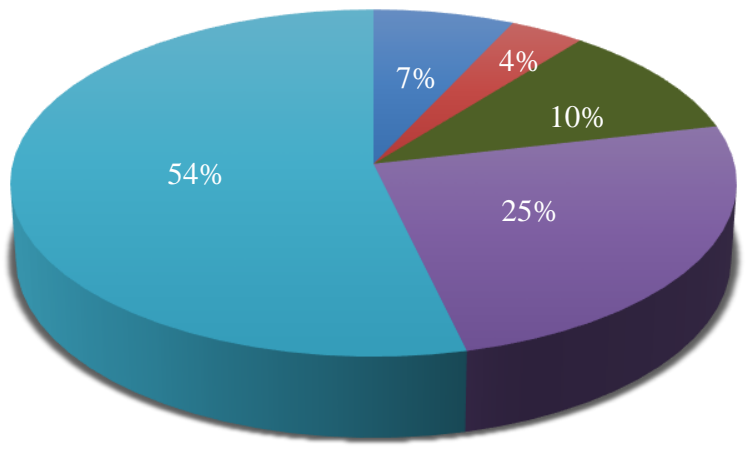

$$
\begin{aligned}
& \text { Strongly disagree } \square \text { Disagree } \\
& \text { Neutral } \quad \text { Agree } \\
& \square \text { Strongly agree }
\end{aligned}
$$

Mobile Health and its Association with Awareness on Non-Communicable Diseases

A majority $(68.7 \%)$ had a health mobile application at the point of the research as shown in Figure 11 and yet $47.83 \%$ rarely used the mobile app actively as shown in Figure 12.
Figure 11: Have You Ever Used Mobile-Based Application?

Have You Ever Used Mobile-Based health Application?

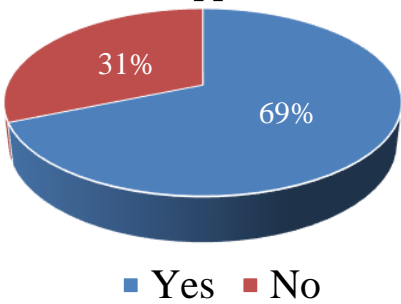

Figure 12: How often the Respondents Use the Mobile-Based Health Application

\section{If, Yes How Often Do You Use It?}

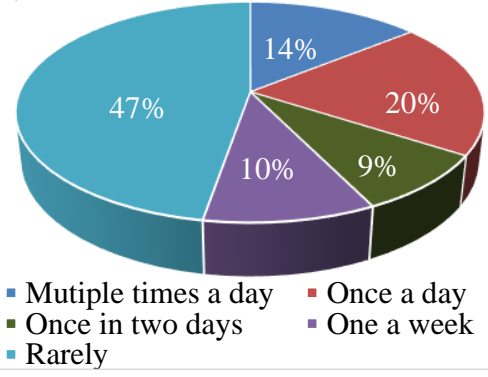

The knowledge on the non-communicable diseases which the participant had, what puts them at risk of non-communicable diseases, and the number of health mobile phone apps that they use was found to be significantly associated with awareness on non-communicable diseases at

$\left(\chi^{2}=10.673 a, d f=3, p=0.001, \chi^{2}=33.282, d f=4\right.$, $p=0.001, \chi 2=38.383, d f=1, p=0.001)$

respectively. The results are as indicated in Table 2.

Table 2: Mobile Health and its Association with Awareness on Non-communicable diseases

\begin{tabular}{llll}
\hline & $\chi^{\mathbf{2}}$ & df & p-value \\
\hline The respondent knows Non-communicable diseases & $7.393^{\mathrm{a}}$ & 2 & 0.362 \\
Which Non-communicable diseases do you know & $10.673^{\mathrm{a}}$ & 3 & $0.001^{*}$ \\
I know what puts me at risk to Non-communicable diseases & 33.282 & 4 & $0.001^{*}$ \\
Non-communicable diseases can be treated & 23.484 & 4 & 0.328 \\
Where did you first get information on Non-communicable diseases & 12.393 & 3 & 0.102 \\
No. of mobile APPs a respondent uses daily & 38.383 & 1 & $0.001^{*}$ \\
No. of hours a respondent spends with a phone & 47.044 & 2 & 0.193 \\
Know any mobile-based app & 29.940 & 2 & 0.329 \\
Whether a mobile phone app can affect health & 17.334 & 1 & 0.536 \\
\hline
\end{tabular}

$\left({ }^{*} \leq \leq 0.05\right.$ is significant at $95 \% \mathrm{Cl}$ ) 


\section{Perception on MyHeart-Ke (mHealth) as a Source of Health Information and Awareness on Non- Communicable Diseases}

Only $18.7 \%$ of the participants deemed MyHeartKe App as not better sources of health information on non-communicable diseases as opposes to the mainstream internet while $52.2 \%$ agreed on MyHeart-Ke being a credible source of health information on NCDs as shown In Figure 13.

Figure 13: MyHeart-Ke is a Source of Information and Awareness on NCDs

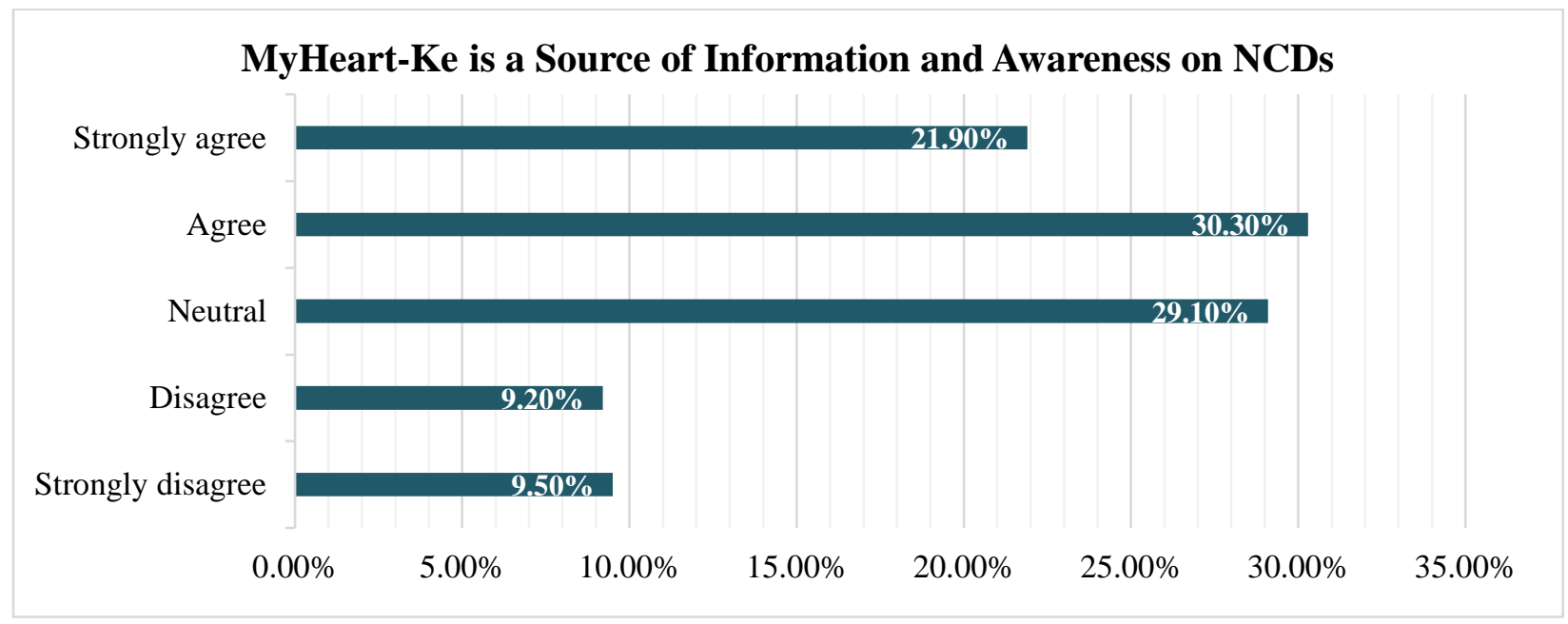

The respondents had varied perceptions on the use of m-Health as a source of information on Noncommunicable diseases. Only whether the mobile apps are a good source of health information was found to be significantly associated with their awareness on non-communicable diseases at

$(\chi 2=36.845, d f=3, p=0.001)$.

The results are as shown in Table 3 below.

Table 3: Perception on the Internet as a Source of Health Information and Awareness on Non communicable diseases

\begin{tabular}{llll}
\hline & $\chi^{2}$ & df & p-value \\
\hline $\begin{array}{l}\text { Internet is a reliable source of information internet may offer misleading } \\
\text { health information }\end{array}$ & 56.443 & 3 & 0.302 \\
$\begin{array}{l}\text { Mobile apps are a good source of health information } \\
\text { Mobile apps generate relevant and easy to understand information }\end{array}$ & 36.845 & 3 & $0.001^{*}$ \\
\hline
\end{tabular}

\section{SUMMARY OF THE FINDINGS AND DISCUSSION.}

\section{Summary of the Findings}

The majority of the study participants were males at $56 \%$ while the majority age was 22 years at $18 \%$. The study found out that awareness on NCDs among the respondents was high at $96 \%$ with cardiovascular diseases being the most widely known at $85.8 \%$. The participants had low knowledge on obesity. The age of the participants was found to be significantly associated with the awareness on NCDs $(\chi 2=34.187, \mathrm{df}=1, \mathrm{p}=0.001)$. On the perceptions, the majority knew the risk factors to NCDs at $89.8 \%$. However, there was a significant number of respondents who were not aware of the risk factors. A high number reported that tobacco use was the major cause of NCDs at $79.9 \%$ while the least indicated that the diseases were genetic and were also caused by poor eating habits at $1 \%$. A majority also had a perception that 
NCDs are preventable through the reduction of alcohol use, screening, and healthy diets at $84 \%$. Most of the participants had learnt about NCDs in school at $75.4 \%$ and they believed that the diseases are a problem among the youths in the country at $48.8 \%$. Although some of the participants had knowledge on m-Health at $68.7 \%$, it was not widely used among the respondents before the study. MyHeart-Ke was reported to offer a significant and relevant amount of information on NCDs with $51.2 \%$ preferring it over the mainstream internet and only $18.7 \%$ not preferring mHealth as better sources of information.

\section{Discussion}

\section{Awareness Level of Non-Communicable Diseases.}

The study found out that awareness on NCDs among the respondents was high with cardiovascular diseases being the most widely known. These findings are in agreement with Boulos et al., (2014) who observes that the youth were knowledgeable on NCDs. Silva et al., (2015) also observed similar findings. The high level of awareness can be attributed to the health campaigns on the need for exercise and good eating habits. Lewis \& Wyatt (2014) note that in countries which advocate for health promotion, the awareness among the populations on most of the health issues is high. The participants had low knowledge on obesity. The findings are not consistent with Silva et al., (2015) who observe that the study participants were knowledgeable about this NCDs. The discrepancies can be attributed to the fact that the study population was different in terms of age. Boulos et al., (2014) undertook the study among all the people aged over 18 years. The age of the participants was found to be significantly associated with the awareness on NCDs. These findings concur with Buijink et al., (2013) who found that the older people were knowledgeable about NCDs. The reason behind this is the misconception that the diseases affect the elderly.

\section{Perception of the Youth towards Non- Communicable Diseases}

The majority knew the risk factors for NCDs. The findings are in line with Ahern et al., (2006) who note that most of the people are knowledgeable about NCDs. A high number reported that tobacco use was the major cause of NCDs while the least indicated that the diseases were genetic and were also caused by poor eating habits. The findings are in agreement with a study which was done by Boulos et al., (2014) who articulate that a majority indicated these are the major predisposing factors to NCDs. A majority also had a perception that NCDs are preventable through the reduction of alcohol use, screening, and healthy diets. The findings concur with Lewis \& Wyatt (2014) who note that a majority of people are aware that NCDs are preventable through medical checks, reduced alcohol usage, and a healthy diet.

\section{Mobile Health and its Association with Awareness on Non-Communicable Disease}

The study found out that the majority were aware of $m$-Health as a source of health information. The findings are in line with Silva et al., (2015) who observe that most of the youths are aware of mobile-based applications that offer health information. The study also noted that a majority of the participants do not use the applications. The findings are in agreement with Buijink et al., (2013) who observe a low uptake of health-based mobile applications among the youths. Although the reasons for low usage were not established, Boulos et al., (2014) note that ignorance, mistrust of the applications, and lack of appealing features in the applications are some of the reasons for their low uptake. However, for MyHeart-Ke App in particular, the study population $51.2 \%$, in fact, agreed that it was a better source of information on cardiovascular diseases and NCDs in general with only $18.7 \%$ disagreeing and this could be attributed to the practicability of the app and the fact that the app provides relevant and simplistic information on NCDs. This could also be attributed to the fact that the app had interesting features including the update tab which app users found very useful especially in linking them to medical camps and new articles on health.

Perception on MyHeart-Ke (mHealth) as a Source of Health Information and Awareness on NonCommunicable Diseases

MyHeart-Ke application was reported to offer a good source of information on NCDs. The findings are in agreement with Ahern et al., (2006) who note that mobile-based applications offer a significant 
knowledge on health matters. Lewis \& Wyatt (2014) also noted similar findings. The study found out that the uptake of the health-based mobile application was slow. These findings concur with Buijink et al., (2013) who found out that despite the awareness that mobile-based application offer, the uptake is slow. Some of the challenges that Neumann (2014) notes are high costs of internet. Although the study did not establish the challenges to the low uptake, this could be among the contributing factors.

\section{CONCLUSION}

The study found out that the majority of the participants were aware of the NCDs as well as some of the predisposing factors after the use of MyHeart-Ke App for 6 months. Cardiovascular disease was among the widely known NCD. The respondents reported tobacco and alcohol use as some of the risk factors to NCDs and most of them understood that the diseases are preventable. Some of the ways of reducing NCDs which they indicated was through reduction of alcohol usage, better diet, and screening. Although some of the participants had knowledge of mHealth, it was not widespread among the respondents. MyHeart-Ke was reported to offer a significant amount of information on NCDs and study participants preferred using it over mainstream internet for information on NCDs.

\section{RECOMMENDATIONS}

\section{Operational Recommendations}

1. The relevant agencies should create more awareness on NCDs among the youths

2. There should be more advocacy on the need to use mobile-based applications as sources of information on NCDs.

\section{Recommendations for Further Research}

1. Research can be undertaken on barriers to mobile-based applications uptake as sources of information on NCDs among the youths

2. A comparative study can be undertaken among urban and rural populations to establish awareness and uptake of mobile-based applications as a source of information on NCDs among the youths

\section{REFERENCES}

Ahern, D. K., Kreslake, J. M., \& Phalen, J. M. (2006). What is eHealth (6): perspectives on the evolution of eHealth research. Journal of medical Internet research, 8(1), e4.

Boulos, M. N. K., Brewer, A. C., Karimkhani, C., Buller, D. B., \& Dellavalle, R. P. (2014). Mobile medical and health apps: state of the art, concerns, regulatory control and certification. Online journal of public health informatics, 5(3), 229.

Breen, G. M., \& Matusitz, J. (2010). An evolutionary examination of telemedicine: a health and computer-mediated communication perspective. Social work in public health, 25(1), 59-71.

Buijink, A. W. G., Visser, B. J., \& Marshall, L. (2013). Medical apps for smartphones: lack of evidence undermines quality and safety. $B M J$ Evidence-Based Medicine, 18(3), 90-92.

Eunice, K. (2017). New partnership to share health advice on phones. Retrieved from https://www.nation.co.ke/health/Newpartnership-to-share-health-advice-onphones/3476990-3893144-fgotavz/index.html

Gorski, I., Bram, J. T., Sutermaster, S., Eckman, M., \& Mehta, K. (2016). Value propositions of mHealth projects. Journal of medical engineering \& technology, 40(7-8), 400-421.

Khf. (n.d.). Website. Retrieved from https://khf.co.ke/how-kenyans-are-embracingmobile-technology-to-access-health-care/

Lee, S., Cho, Y. M., \& Kim, S. Y. (2017). Mapping mHealth (mobile health) and mobile penetrations in sub-Saharan Africa for strategic regional collaboration in mHealth scale-up: An application of exploratory spatial data analysis. Globalization and health, 13(1), 63.

Lewis, T. L., \& Wyatt, J. C. (2014). mHealth and mobile medical apps: a framework to assess 
risk and promote safer use. Journal of medical Internet research, 16(9), e210.

Maina, D. W. (2019). Perspectives of Patients with Comorbidity on the Use of eHealth Technology for Self-Management at Kenyatta National Hospital.

Mburu, S., \& Oboko, R. (2018). A model for predicting utilization of mHealth interventions in low-resource settings: Case of maternal and newborn care in Kenya. BMC medical informatics and decision making, 18(1), 67.

NCD mortality and morbidity. (2018, August 24). Retrieved from https://www.who.int/gho/ncd/mortality_morbi dity/en/

NCD and Youth. (2016, July 4). Retrieved from https://www.who.int/global-coordinationmechanism/ncd-themes/ncd-and-youth/en/

Neumann, M. M. (2014). An examination of touch screen tablets and emergent literacy in Australian pre-school children. Australian Journal of Education, 58(2), 109-122.

Njoroge, M., Zurovac, D., Ogara, E. A., Chuma, J., \& Kirigia, D. (2017). Assessing the feasibility of eHealth and mHealth: a systematic review and analysis of initiatives implemented in Kenya. BMC research notes, 10(1), 90.

Reynolds, K. (2017). Evaluating Caller Experience with a Telephone Health Hotline in Malawi (Doctoral dissertation).

Shiroya, V., Neuhann, F., Müller, O., \& Deckert, A. (2019). Challenges in policy reforms for noncommunicable diseases: the case of diabetes in Kenya. Global Health Action, 12(1), 1611243. doi: 10.1080/16549716.2019.1611243

Silva, B. M., Rodrigues, J. J., de la Torre Díez, I., López-Coronado, M., \& Saleem, K. (2015). Mobile-health: A review of current state in 2015. Journal of biomedical informatics, 56, 265-272. 